\title{
XELOX Regimen
}

National Cancer Institute

\section{Source}

National Cancer Institute. XELOX Regimen. NCI Thesaurus. Code C63597.

A regimen consisting of capecitabine and oxaliplatin used for the treatment of advanced stage colorectal cancer. This regimen differs from a similar regimen, CAPOX, with regards to the dosing schedule for oxaliplatin. 